\title{
Effects of retraining following response prevention on extinction of escape behavior
}

\author{
JOSEPH J. FRANCHINA, TIMOTHY E. SCHINDELE, and KAREN R. RITENOUR \\ Virginia Poly'technic Institute and State University, Blacksburg, Virginia 24061
}

\begin{abstract}
Sixty female hooded rats received a sequence of 40 shockescape training trials, 20 response prevention (or pseudoprevention) trials, either 0,5 , or 20 shock-escape retraining trials and then nonshock extinction procedures. Results of extinction using escape speed and trials-to-criterion indices showed that, in each retraining condition, response prevention reliably facilitated extinction relative to pseudoprevention controls, the degree of facilitation decreasing as amount of retraining increased. In 5and 20-trial retraining conditions, prevention procedures also reliably impaired shock-escape performance on early retraining trials, this effect dissipating before the start of extinction.
\end{abstract}

Response prevention (blocking) facilitates extinction of previously established avoidance or escape behavior (e.g., Baum, 1970; Franchina, Agee, \& Hauser, 1974). Prevention procedures essentially consist in the confinement of a subject (usually a rat) in a situation where shock previously occurred, confinement conditions precluding the presentation of shock and the opportunity to perform the previously established instrumental response (cf. Baum, 1970; Solomon, Kamin, \& Wynne, 1953, Experiment 3). The effects of prevention are then typically measured by subsequent performance in extinction under nonshock conditions.

Since prevention procedures are intended to curtail the influence of prior aversive training, the effectiveness of prevention in facilitating extinction has been evaluated mainly as a function of training parameters, like shock intensity, or prevention parameters, like prevention duration. Recently, however, prevention's effectiveness has been assessed by studying extinction performance following the interpolation of retraining/reconditioning trials between prevention procedures and subsequent extinction responding. For example, Franchina et al. (1974, Experiment 3) reported that interpolating one shock-escape retraining trial between prevention procedures and subsequent extinction attenuated, but did not eliminate, a reliable prevention effect. Bersh and Keltz (1971) reported that interpolating one Pavlovian conditioning trial of inescapable shock between two successive extinction sessions eliminated the effects of prevention on extinction for all but the longest prevention condition.

These studies indicate that the influence of prevention persisted sufficiently to facilitate the extinction of a previously established instrumental response following the interpolation of shock-retraining procedures, extinction being measured under nonshock conditions.

Requests for reprints should be sent to J. J. Franchina, Department of Psychology, Virginia Polytechnic Institute and State University, Blacksburg, Virginia 24061. Thanks are due to R. A. Hughes for a critical reading of this report.
However, the results of Bersh and Keltz (1971) and Franchina et al. (1974) constitute somewhat limited information. Their use of one retraining/reconditioning trial provided only a very brief reinstatement of original shock conditions and, in fact, only Franchina et al. (1974) administered retraining between prevention procedures and instrumental response extinction procedures. Further, Franchina et al. (1974) reported that, in retraining, escape performance was reliably lower for the prevention group than for nonprevention controls, a finding which suggests that one retraining trial differentially affected shock-escape performance for prevention and nonprevention conditions. Thus, the effects of prevention on subsequent performance in nonshock extinction might have been mitigated by a confounding of prevention conditions with level of responding prior to extinction.

To provide further information on the durability of prevention's influence through the use of retraining procedures, the present study investigated the effects of prevention on nonshock extinction performance following the interpolation of 0,5 , or 20 shock-escape retraining trials between prevention procedures and subsequent extinction. Since Franchina et al. (1974) suggest that prevention procedures may affect subsequent shock-escape responding in retraining, as well as affecting later performance in nonshock extinction, multiple retraining trials ( 5 or 20 ) were administered to obtain information on the possible influence of prevention on retraining performance per se and to provide that, at each retraining level, prevention and nonprevention groups perform similarly to each other prior to extinction.

\section{METHOD}

\footnotetext{
Apparatus

The apparatus was that described by Franchina, Bush, Kash, Troen, and Young (1973, Experiment 3). Briefly, a shock box was separated from a safe box by a guillotine door and a hurdle The boxes were made of wood and appeared highly similar to each other. In each box, the walls were covered with white
} 
Plexiglas; the floor were grid rods; the illumination level was always $110 \mathrm{fc}$. The grid floor of the shock box was connected to a constant voltage shock $(\mathrm{dc})$ source. The current measured at the grids was $1.0 \mathrm{~mA}$. The safe-box floor was movable and could be depressed by the rat's weight to act as a switch.

\section{Subjects}

Sixty experimentally naive female hooded rats, 110-130 days old, were obtained from the local departmental colony. The rats were housed in pairs with food and water ad lib throughout the experiment.

\section{Design and Procedure}

Each rat received this sequence: Day 1 -handling and 6 min of apparatus exploration; Day 2 -forty shock escape training trials; Day 3-20 response prevention trials or 20 pseudoprevention trials; Day 4-0, 5, or 20 shock-escape retraining trials; Days 5-8 -40 nonshock extinction trials per day to an extinction criterion or to a 160-trial limit. The design of this experiment was a 2 by 3 factorial, orthogonally combining two prevention conditions, RP and $\overline{\mathrm{P}}$, with three levels of retraining, 0,5 , or 20 trials.

For a shockescape training trial, the rat was placed into the shock box; $5 \mathrm{sec}$ later, the guillotine door was raised, simultaneously activating the shock and a timer, calibrated in $.01 \mathrm{sec}$. If the rat jumped the liurdle within $40 \mathrm{sec}$, the safe-box floor was depressed, tuming off the shock and timer. After $20 \mathrm{sec}$ in the safe box, the rat was removed manually to an unpainted wooden holding box $(30 \times 30 \times 30 \mathrm{~cm})$ for an intertrial interval (ITI) of 30 to $60 \mathrm{sec}$. If the rat failed to jump the hurdle within $40 \mathrm{sec}$, the guillotine door was closed; shock remained on; and the rat was removed to the holding box for the ITI. After training, the rats were randomly assigned to groups $(\mathrm{N}=30$ ) for response prevention $(\mathrm{RP})$ or pseudoprevention $(\overline{\mathrm{P}})$ procedures which were administered on the next day.

For an RP trial, the rat was placed into the shock box; after $5 \mathrm{sec}$, the guillotinc door was raised, activating the timer but no shock occurred. A clear Plexiglas barrier blocked entry in to the sate box. After an additional $15 \mathrm{sec}$, the rat was removed manually to the holding box for an ITI of $30 \mathrm{sec}$. For a $\overline{\mathrm{P}}$ trial, the rat was placed into a wire cage for $20 \mathrm{sec}$. The cage was $30 \times 30 \times 30 \mathrm{~cm}$. It was constructed of $2.57 \mathrm{~cm}$ hardware cloth and had a cardboard top. The wire cage was located $8.28 \mathrm{~cm}$ from the side of the shock box. After $20 \mathrm{sec}$ of confinement, the rat was removed to the holding box for the ITI. After the end of Day 3 procedures, the rats in $R P$ and $\overline{\mathrm{P}}$ conditions were randomly assigned to groups $(\mathrm{N}=20)$ for 0,5 , or 20 retraining trials administered on the next day.

On the retraining day (Day 4), all rats were brought in to the experimental room. Since the different numbers of retraining trials would provide for differential amounts of handling, each rat was handled 20 times. This total consisted of handling due to the assigned number of retraining trials, 0,5 , or 20 , plus a complementary number of handlings, 20,15 , or 0 , unrelated to training. The latter consisted in lifting the rat from the holding box, placing the rat on the experimenter's arm for $20 \mathrm{sec}$, and then, returning the rat to the holding box for the ITI. These handling procedures preceded retraining and provided that each rat was in the experimental room for 15 to $20 \mathrm{~min}$. Retraining trial procedures and ITI were those of original training.

Beginning on the next day (Day 5), nonshock extinction trials were administered at 40 trials per day until an extinction criterion or a limit of 160 trials was reached. The extinction criterion was the rat's failure to leave the shock box within $10 \mathrm{sec}$ on four of five consecutive trials. However, to provide an index of hurdle-jump performance over a uniform number of extinction trials, 30 extinction trials were administered to all rats on the first extinction day (Day 5), regardless of whether the rat at tained the extinction criterion. If the extinction criterion was attained within 30 trials, then extinction was terminated immediately following the 30 th trial. If not, then extinction continued to sriterion or to a total of 160 trials, whichever came first. Extinction trial procedures were the same as those of training trials, except that shock was omitted. In original training, retraining, and extinction, the performance measure was hurdle-jumping latency which was converted into reciprocal scores.

\section{RESULTS}

Figure 1 presents mean reciprocals of latency of hurdle jumping for the last two blocks of original shock-escape training, for shock-escape retraining, and for the first three trial blocks of nonshock extinction. Terminal performance in original training showed no reliable group effects $(\mathrm{F}<1)$.

For retraining, Figure 1 shows that shock-escape performance was higher for the 20. than for the 5-retraining-trial groups in $\mathrm{RP}$ and $\overline{\mathbf{P}}$ conditions, the magnitude of this effect being greater in RP. Interestingly, over Trials 1-5 (not shown), for the

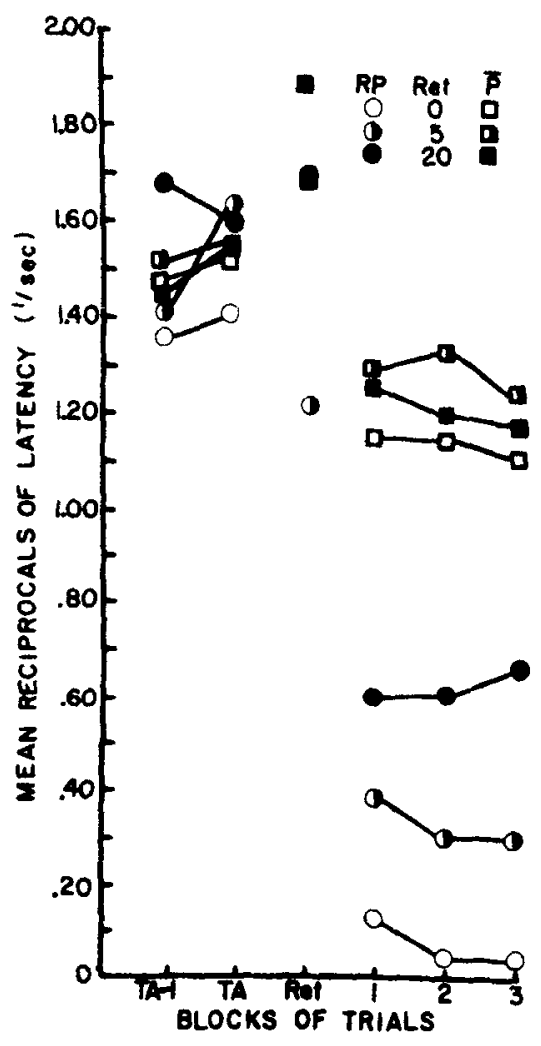

Figure 1. Mean reciprocals of latency of hurdle jumping in blocks of 10 trials for the last two blocks of original training (TA-1, TA) and for blocks 1,2 , and 3 of extinction for RP and $\bar{P}$ groups that received 0,5 , or 20 retraining trials. The unconnected points following original training show mean reciprocals of latency on Trials 1 through 5 for the 5 -retraining-trial group and Trials 16 through 20 for the 20 -retraining-trial group, $R P$ and $\overline{\mathbf{P}}$ conditions. ( $\mathrm{RP}$-circle symbols; $\overline{\mathrm{P}}$-square symbols; 0 Retraining trials-open symbols; 5 Retraining trials-half-filled-in symbols; 20 Retraining trials-completely filled-in symbols.) 
20-trial group, mean reciprocals of latency were 1.12 and 1.64 for RP and $\bar{P}$ conditions, respectively. These mean scores are highly similar to those over Trials $1-5$ for the five-trial groups, $\mathrm{RP}$ and $\overline{\mathrm{P}}$ conditions, shown in Figure 1. Since the main concern of this experiment was extinction performance, a more detailed presentation of shock-escape performance in retraining will be shown after the extinction results.

For extinction, Figure 1 shows that prevention procedures facilitated extinction under nonshock following each level of shockescape retraining, the magnitude of this effect declining with increasing retraining. That is, the difference in extinction performance between $R P$ and $\overline{\mathrm{P}}$ conditions decreased as number of retraining trials increased. This variation in prevention effects seems due to the relatively greater enhancement of extinction performance for the RP than for the $\bar{P}$ condition following retraining. Analysis of variance of the extinction data of Figure 1 yielded reliable effects for amount of retraining, prevention conditions, and their interaction $(\mathrm{F}=3.93,63.77$, $16.81 ; \mathrm{df}=2 / 54,1 / 54,2 / 54 ; \mathrm{p}<.05,<.001,<.001$, respectively). Simple effects analyses for the reliable interaction showed that amount of retraining was reliable for $\mathrm{RP}(\mathrm{p}<.001)$ but not for $\overline{\mathrm{P}}(\mathrm{F}=1.27)$. The difference between $R P$ and $\bar{P}$ conditions was reliable at each retraining level ( $\mathrm{ps}<.01$ ).

Considering the results for the trials-to-criterion index of extinction, mean trials to criterion for $0-, 5-$, and 20-retraining-trial groups in the RP condition were 5.4, 23.6, and 63.6, respectively, and in the $\overline{\mathrm{P}}$ condition, 79.6, 92.0, and 95.9, respectively. (These scores included criterional trials.) These data were essentially consistent with the reciprocal of latency data. Prevention facilitated extinction following each level of retraining, the degree of facilitation decreasing with increasing retraining. Analysis of variance of the trials-to-criterion data yielded reliable effects for prevention condition, amount of retraining, and their interaction $(\mathrm{F}=17.62,5.75,11.73 ; \mathrm{df}=1 / 54,2 / 54$, $2 / 54 ; \mathrm{p}<.001,<.01,<.001$, respectively). Simple effects analyses of the reliable interaction yielded results highly consistent with those of analyses of the reciprocal of latency data.

Considering retraining, Figure 2 shows mean reciprocals of latency on each trial for Trials 1 through 5 of the 5-retraining-trial group and on each trial for Trials 16 through 20 of the 20 -retraining-trial group, RP and $\bar{P}$ conditions. Figure 2 does not show shock-escape performance for Trials 1 through 5 (i.e., early retraining) of the 20-retraining-trial group, RP and $\overrightarrow{\mathrm{P}}$ conditions. However, the evaluate performance between the latter retraining group and the five-trial retraining group over the same stage of retraining (i.e., Trials 1-5), the results of each trial of Trials 1 through 5 are herewith presented for the 20-retraining-trial group: Mean reciprocals of

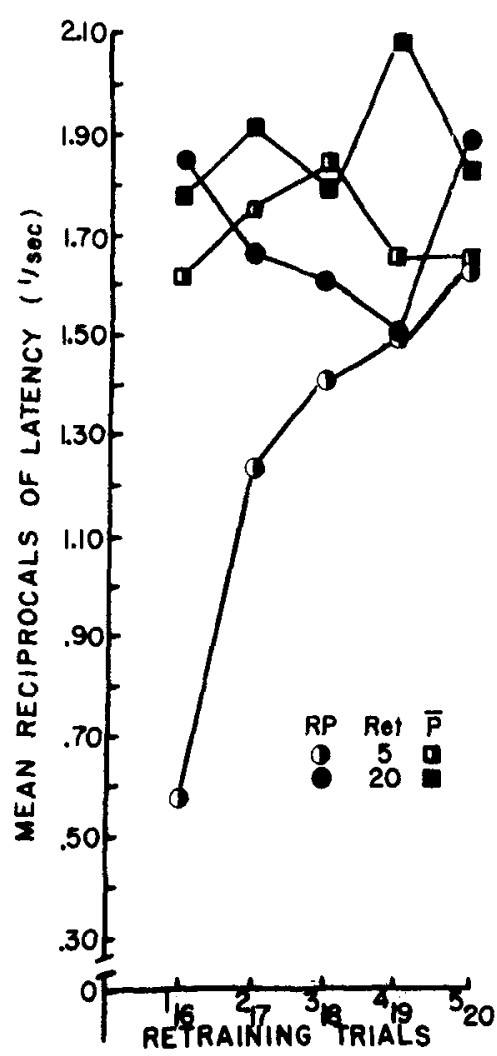

Figure 2. Mean reciprocals of latency of hurdle jump in retraining on each trial of Trials 1 through 5 for the 5-retraining-trial group and on each trial of Trials 16 through 20 for the 20-retraining-trial group, $R P$ and $\bar{P}$ conditions. (RP-circle symbols; $\overline{\mathrm{P}}$-square symbols; Trials 1 through 5-5-trial group-half- filled-in symbols; Trials 16 through 20-20-trial group-completely filled-in symbols.)

latency for the RP condition were $.35,1.26,1.40,1.60$, and 1.58 , and for the $\overline{\mathrm{P}}$ condition, $1.38,1.70,1.54$, 1.55, and 1.75. A comparison between these data and those of the five-trial groups in Figure 2 shows that, in early retraining (Trials $1-5$ ), performance of the 20-trial group was highly similar to that of the 5 -trial group in $\mathrm{RP}$ and $\overline{\mathrm{P}}$ conditions.

Considering Trials 1 through 5 , the results of the 5-trial group in Figure 2 and those of the 20-trial group (just presented) show that on Trials 1,2 , and 3 performance was poorer for RP than for $\overline{\mathbf{P}}$ conditions. This effect dissipated with retraining, however, and Trials 4 and 5 show that $R P$ and $\bar{P}$ conditions performed similarly to each other. Analysis of variance comparing 5- and 20-trial groups on Trials 1 through 5 only, RP and $\overline{\mathbf{P}}$ conditions, yielded reliable effects for prevention conditions and Trials by Prevention Condition $(F=19.28$, and $2.84 ; \mathrm{df}=1 / 36$ and $4 / 144 ; \mathrm{p}<.001$ and $<.05$, respectively). No reliable effect was obtained for the dummy variable, amount of retraining, in this analysis $(F=.79$.Simple effects analyses for the reliable interaction showed that RP and $\overline{\mathrm{P}}$ conditions differed 
reliably $(p<.05)$ from each other on retraining Trials 1 , 2 , and 3 but not on Trials 4 or $5(\mathrm{p}>.20)$.

In Figure 2, comparison of Trials 16 through 20 for the 20-trial groups and Trials 1 through 5 for the 5-trial groups shows that the greater the number of retraining trials, the higher the shock-escape performance in both $\mathrm{RP}$ and $\overline{\mathrm{P}}$ conditions, this enhancement of performance heing greater for RP. For the 20-trial group, the difference between $R P$ and $\bar{P}$ conditions appears to be inconsistent and, for the most part, negligible as compared to the marked RP. $\overline{\mathrm{P}}$ difference for the 5-trial group. Analysis of variance over all the data of Figure 2 compared Trials 1 through 5 of the 5 -trial group with Trials 16 through 20 of the 20-trial group, RP and $\bar{P}$ conditions. (This analysis did not consider Trials 1 . through 5 of the 20-trial group.) Reliable effects were obtained for amount of retraining, prevention condition, Retraining by Prevention condition, and Trials by Prevention condition $(\mathrm{F}=4.96,5.02,14.23,2.52$; $\mathrm{df}=1 / 36,1 / 36,1 / 36,4 / 144 ; \mathrm{p}<.05,<.05,<.001$, $<.05$, respectively). Simple effects analyses showed that the Trials by Prevention Condition interaction was reliable for the five-trial group $(p<.05)$. Neither the effect of prevention condition nor the interaction of Trials by Prevention Condition was reliable for the 20-trial group $(\mathrm{F}=2.00,1.73 ; \mathrm{df}=1 / 36,4 / 144$; $p>.10)$. The reliable interaction of Trials by Prevention Condition for the five-trial group was due to reliable differences between RP and $\bar{P}$ on Trials 1,2, and 3 but not on Trials 4 or 5 . Comparisons between the 5-trial group (Trials 1-5) and the 20-trial group (Trials 16-20) for $\mathrm{RP}$ and for $\overline{\mathrm{P}}$ conditions separately showed that the effect of number of retraining trials was reliable for RP $(\mathrm{p}<.01)$ but not for $\overline{\mathrm{P}}(\mathrm{F}=.86)$. These findings provide statistical support for the results of retraining shown in Figures 1 and 2: Response prevention reliably impaired shock-escape performance on initial retraining trials. Number of retraining trials enhanced shock-escape performance more following response prevention than following pseudoprevention procedures.

\section{DISCUSSION}

This experiment shows that response prevention reliably facilitates the subsequent extinction of escape behavior under nonshock conditions despite reinstatement of shock-escape training between prevention and extinction procedures. However, prevention's facilitation of later extinction decreases reliably as the amount of intervening retraining increases. The present results are consistent with those of Franchina et al. (1974, Experiment 3). Together, these studies suggest that the influence of prevention persists sufficiently through shock-retraining procedures to affect later extinction. Unlike the data of Franchina et al. (1974, Experiment 3) the current results do not seem susceptible to criticism based on a potential confounding between prevention conditions and level of shock-escape responding prior to the start of extinction. In the present experiment, $R P$ and $\bar{P}$ conditions performed similarly to each other at the end of 5- and 20-retraining trials (see Figure 2). If the absence of reliable differences between $R P$ and $\overline{\mathrm{P}}$ in terminal retraining reflects similar strengths of instrumental responding between these conditions, then the RP. $\bar{P}$ differences in extinction are not likely attributable to a confounding of prevention conditions with terminal retraining levels. It might be countered that the similarity of terminal retraining performance between $R P$ and $\bar{P}$ was due to occurrence of a physiological limit in responding by $\overline{\mathrm{P}}$ condition. Statistical analyses and Figure 1 do indicate that $\overline{\mathbf{P}}$ condition's terminal level of responding did not increase reliably with increasing retraining trials. However, previously reported data from Franchina (1969) and Franchina et al. (1973) raise some doubts about the influence of a physiological limit in the present results. In the latter studies, shock-escape training procedures, similar to those of this experiment, yielded pe:formance levels in excess of those reported here. Finally, whatever confounding factors may taint the present reciprocals of latency data, this contamination seems unlikely for the trials-to-criterion data. The latter are essentially parallel to the reciprocal of latency results.

The finding of reliable prevention effects following unreliable RP. $\overline{\mathrm{P}}$ differences in terminal retraining is difficult to interpret from current prevention hypotheses (see Baum, 1970). For example, the competing response hypothesis (Coulter, Riccio, \& Page, 1969) proposes that prevention provides for adventitious reinforcement of instrumental behaviors (e.g., sitting/freezing) which are antagonistic to previously trained avoidance/escape behavior. Subsequent elicitation of these acquired competing responses in nonshock extinction may curtail avoidance/escape performance and thereby facilitate extinction. In the present experiment, competing responses acquired during prevention may have persisted into retraining where their occurrence in the shock box on retraining trials was presumably punished by shock presentation. Punishment of competing responses in retraining should then have decreased the likelihood of competing response elicitation in later extinction. Further, shock offset after escape responding in retraining should have provided reinforcement for the originally trained instrumental response. Since reinforcement should facilitate the reemergence of escape behavior, elicitation of antagonistic competing responses should, conversely, be curtailed and be less influential in later extinction. Finally, since RP and $\bar{P}$ conditions did not differ reliably from each other in terminal retraining, compelling evidence of the availability of competing responses seems lacking prior 
to the start of extinction. Considering this factor and the previously mentioned effects of retraining on acquired competing responses, interpretation of the present extinction results by the competing response hypothesis seems difficult.

The present results could be construed as being consistent with the fear-extinction hypothesis of prevention (see Baum, 1970). Prevention provides exposure to shock-box stimuli which previously accompanied shock in training. Since stimulus exposure occurs under nonshock conditions, fear, established to these cues by prior shock, should be decreased following prevention and the instrumental response based on prior fear should decline (e.g., in later extinction). In the present study, prevention procedures presumably reduced the level of fear for $R P$ below that of $\bar{P}$ conditions. If one assumes that this difference in fear was relatively unaffected by application of a constant number of retraining trials ( 5 or 20 ) for $R P$ and $\bar{P}$, then the present results may reflect the influence of less fear for RP than for $\overline{\mathbf{P}}$ conditions. A problem for this interpretation, however, may be in the reasonableness of assuming that the difference in levels of fear between RP and $\overline{\mathrm{P}}$ conditions remains relatively stable through retraining. Increasing numbers of shock retraining trials (up to 20 in this study) should increase the strength of fear for both $\mathrm{RP}$ and $\overline{\mathrm{P}}$ conditions toward some asymptote. This trend, in turn, could conceivably reduce the degree of difference in levels of fear between conditions. Further, present procedures do not permit evaluation of the effects of fear separate from those of instrumental performance per se. Consequently, a firm conclusion about the role of fear in prevention and, now, in retraining must await the results of such evaluations (Coulter et al., 1969).

Finally, this experiment shows that prevention procedures not only facilitate extinction of escape behavior under nonshock extinction conditions but also impair initial shock-escape performance on shock-retraining trials. Interpretation of these data may come from either competing response or fear-extinction hypotheses of prevention although neither hypothesis has been explicitly directed toward explaining shock-escape/avoidance performance subsequent to prevention procedures (Baum, 1970). Nevertheless, the acquisition of some postural response during prevention or the lowering of the level of fear for RP conditions following prevention could account for the temporary impairment of escape responding for $\mathrm{RP}$ relative to $\overline{\mathrm{P}}$ on early retraining trials. An interesting implication of these data is the suggestion that prevention may attenuate the effectiveness of subsequent as well as prior aversive training.

\section{REFERENCES}

Baum, M. Extinction of avoidance responding through response prevention (Flooding). Psychological. Bulletin, 1970, 74, 275-284

Bersh, P. J., \& Keltz, J. R. Pavlovian reconditioning and the recovery of avoidance behavior in rats after extinction with response prevention. Journal of Comparative and Physiological Psychology, 1971, 76, 262-266.

Coulter, X., Riccio, D. C., \& Page, H. A. Effects of blocking an instrumental avoidance response: Facilitated extinction but persistence of "fear." Journal of Comparative and Physiological Psychology, 1969, 68, 377-381.

Franchina, J. J. Escape behavior and shock intensity : Within-subject versus between-group comparisons. Journal of Comparative and Physiological Psychology, 1969, 69, 241-245.

Franchina, J. J., Agee, C. M., \& Hauser, P. J. Response prevention and extinction of escape behavior: Duration frequency, similarity and retraining variables. Journal of Comparative and Physiological Psychology, 1974, 87. 354-363.

Franchina, J. J., Bush, M. E., Kash, J. A., Troen, D. M., \& Young, R. L. Similarity between shock and safe areas during acquisition, transfer, and extinction of escape behavior in rats. Journal of Comparative and Physiological Psychology, 1973, 84, 216-224.

Solomon, R. L., Kamin, L. J., \& Wynne, L. C. Traumatic avoidance learning: The outcome of several extinction procedures with dogs. Journal of Abnormal and Social Psychology, 1953, 48, 291-302.

(Received for publication August 13, 1974; revision accepted January 7,1975 .) 